\title{
Intraoperative photoacoustic screening of breast cancer: a new perspective on malignancy visualization and surgical guidance
}

Ivan Kosik

Muriel Brackstone

Anat Kornecki

Astrid Chamson-Reig

Philip Wong

Morteza Heydari Araghi

Jeffrey J. L. Carson 


\title{
Intraoperative photoacoustic screening of breast cancer: a new perspective on malignancy visualization and surgical guidance
}

\author{
Ivan Kosik, ${ }^{a, b, *}$ Muriel Brackstone, ${ }^{c, d}$ Anat Kornecki, ${ }^{a, e}$ Astrid Chamson-Reig, ${ }^{a}$ Philip Wong, ${ }^{a, b}$ \\ Morteza Heydari Araghi, ${ }^{a}$ and Jeffrey J. L. Carson ${ }^{\mathrm{a}, \mathrm{b}, \mathrm{d}}$ \\ aLawson Health Research Institute, Imaging Program, London, Ontario, Canada \\ ${ }^{b}$ Schulich School of Medicine and Dentistry, Department of Medical Biophysics, London, Ontario, Canada \\ 'Schulich School of Medicine and Dentistry, Department of Oncology, London, Ontario, Canada \\ ${ }^{d}$ Schulich School of Medicine and Dentistry, Department of Surgery, London, Ontario, Canada \\ eSchulich School of Medicine and Dentistry, Department of Medical Imaging, London, Ontario, Canada
}

\begin{abstract}
High re-excision rates in breast-conserving surgery call for a new intraoperative approach to the lumpectomy margin evaluation problem. The unique intraoperative imaging system, presented here, demonstrated the capability of photoacoustic tomography (PAT) to deliver optical sensitivity and specificity, along with over 2-cm imaging depth, in a clinical setting. The system enabled the evaluation of tumor extent, shape, morphology, and position within lumpectomy specimens measuring up to $11 \mathrm{~cm}$ in diameter. The investigation included all major breast cancer-related lesions, such as invasive ductal carcinoma (IDC), multifocal IDC, ductal carcinoma in situ and combinations of these variants. Coregistration with established ultrasound (US) technology, as well as comparison to specimen radiography, validated the performance of PAT, which appeared to facilitate better tumor visualization. Contrary to expected PA contrast mechanisms, PAT images of hemoglobin distribution correlated poorly with US-determined tumor location, while hypointense regions in lipid-weighted PAT images were in better agreement with US. ๑ The Authors. Published by SPIE under a Creative Commons Attribution 4.0 Unported License. Distribution or reproduction of this work in whole or in part requires full attribution of the original publication, including its DOI. [DOI: 10.1117/1 .JBO.24.5.056002]
\end{abstract}

Keywords: photoacoustic; breast cancer; surgical guidance; three-dimensional; tomography.

Paper 180595RR received Oct. 29, 2018; accepted for publication Apr. 23, 2019; published online May 20, 2019.

\section{Introduction}

\subsection{Overview}

The past few decades have witnessed the introduction of advanced imaging methods, regular screening programs, and therapies causing breast cancer survival rates to improve significantly; however, it still remains as the most common cancer to affect women, excluding basal cell carcinoma. Today, it is estimated that about $25 \%$ of new cancer cases in women will be breast cancer, resulting in $15 \%$ of all cancer mortalities. ${ }^{1}$

Typically, treatment of stages I and II diagnoses of breast cancer includes breast-conserving surgery (BCS), sometimes referred to as lumpectomy. The goal is to remove all malignant tissues from the breast surrounded by a continuous envelope of normal tissues. This healthy tissue layer is referred to as a negative margin. ${ }^{2}$ Currently, selection of the width of a lumpectomy margin to minimize cancer recurrence is a topic of debate. Opinions range from $<2 \mathrm{~mm}$ to $>10 \mathrm{~mm}$ depending on age, cancer type, and therapies employed..$^{2-4}$ Nevertheless, in current clinical practice, the procedure often fails, resulting in a positive margin being reported by pathology at a later time and leading to a national (Canadian) re-excision rate of $23 \% .^{5,6}$ To minimize the risk of cancer recurrence, such a finding usually leads to the need for a second surgery. This situation has a negative impact on hospital resources and patients, by delaying adjuvant therapy, elevating stress levels, and degrading cosmetic outcome. For

*Address all correspondence to Ivan Kosik, E-mail: ivalus@uwo.ca example, a recent retrospective review of data for nearly 10,000 patients in the United States found that re-excision was associated with an average additional cost of $\$ 16,072$ along with twice the likelihood of complications such as infection, fat necrosis, or hematoma, compared to patients with no reexcision ${ }^{7}$. Furthermore, another study of 6622 cases found that just one re-excision delayed adjuvant therapy by 2.1 weeks, while a third added 5.7 weeks. ${ }^{8}$ Reducing BCS re-excision rates would translate to a substantial improvement in breast cancer care from both a monetary perspective and personal perspective.

Clearly, a supplemental intraoperative margin assessment method could be of great benefit if it is able to quickly detect and visually guide the operating surgeon toward missed malignant tissue. There are several techniques that have been investigated. For example, frozen sectioning allows for visual inspection of the margin but requires special expertise, is time-consuming, and can be difficult to interpret due to artifacts caused by the freezing process. ${ }^{9}$ Intraoperative contact cytology reduces the processing time but is limited to surface examination and suffers from variable sensitivity related to pathologist skill level. ${ }^{10}$ Specimen radiology is convenient but offers poor contrast, especially in denser breasts, resulting in poor correlation with histological measurements. ${ }^{11}$ Of note is a recently FDAapproved device called the MarginProbe ${ }^{\mathrm{TM}}$, which uses radio frequency spectroscopy to interrogate a 7-mm diameter area of interest to a depth of up to $1 \mathrm{~mm}$. On the downside, limited sensitivity, specificity, field of view, and penetration imply that complete margin assessment is neither practical nor possible, leading to a possibility of missed malignant tissue within the 
margin. ${ }^{12}$ Intraoperative ultrasound (US) examination is a convenient, nondestructive method with reported high specificity; however, it remains unable to overcome sensitivity problems in younger and denser breasts especially with high-grade tumors exhibiting intraductal components. ${ }^{13,14}$

Alternatively, recent studies using optical techniques, such as Raman spectroscopy, autofluorescence spectroscopy, optical coherence tomography, and diffuse reflectance spectroscopy have demonstrated superior soft tissue contrast, resulting in margin assessment sensitivity as high as $100 \%$ and specificity of nearly $92 \% .^{15-19}$ Unfortunately, the desirable diagnostic properties of these methods are offset by either their limited penetration depth or insufficient resolution. A margin evaluation method encompassing the specificity and sensitivity of optical imaging but with deep tissue penetration (i.e., $>10 \mathrm{~mm}$ ) and sufficient resolution would therefore offer substantial advantages over currently employed techniques.

Photoacoustic tomography (PAT) has recently been used to demonstrate highly desirable capabilities in the arena of ex vivo and in vivo soft tissue visualization. The success of this technique is in large part due to its ability to sway imaging contrast toward specific tissue chromophores, such as oxyhemoglobin, deoxyhemoglobin, lipid or calcium oxalate, and hydroxyapatite (i.e., microcalcifications). ${ }^{20-22}$ Furthermore, PAT overcomes limited depth penetration by ultrasonically encoding deep tissue optical contrast and, therefore, similar to diagnostic US, is able to achieve several centimeters of penetration and near millimeter, or submillimeter, resolution. ${ }^{23-25}$

In spite of these favorable properties, PAT has rarely been evaluated for surgical intervention and only recently for assessing breast lumpectomy margins. In a study performed at Indiana University, 12 lumpectomy tissue specimens were assessed by employing a commercially available hybrid US and PA imaging system with reported $100 \%$ sensitivity and $75 \%$ specificity. ${ }^{26}$ However, the investigation included a small number of samples and only invasive ductal carcinoma (IDC) lesions, raising concerns over statistical significance as well as ability to evaluate other types of tumors, such as ductal carcinoma in situ (DCIS) and invasive lobular carcinoma, which currently represent a substantial clinical problem. ${ }^{27-29}$ Furthermore, depending on the standard of practice employed in a particular region or country, the reported 3-mm imaging penetration may or may not be enough to examine the margin to sufficient depth. ${ }^{3,4,27}$ From a technical perspective, the limited view reflection mode imaging geometry employed in that study, combined with a highfrequency transducer, invites well-documented PA imaging problems such as limited view and reflection artifacts, and shallow tissue penetration. ${ }^{30,31}$ And finally, the study utilized BCS specimens that were fixed in $10 \%$ formalin as well as embedded in $2.5 \%$ gelatin; these both are time-consuming procedures and hence unrealistic with respect to clinical intraoperative applications. Extension of the results should, therefore, be made with caution since the effect of sample preparation on sensitivity and specificity is unknown. Nevertheless, the results were highly encouraging, highlighting the potential of a dedicated intraoperative lumpectomy margin evaluation system based on PAT.

\subsection{Objectives}

Here we report on the results from BCS patients using a custombuilt intraoperative photoacoustic screening (iPAS) system. The results include all major breast cancer-related lesions such as IDC, DCIS, multifocal, IDC with extensive intraductal component, and benign, nonpalpable, and occult lesions. The results provide an evaluation of the capabilities of iPAS to assess the extent and composition of breast abnormalities during lumpectomy surgery.

\section{Materials and Methods}

\subsection{Intraoperative Photoacoustic Screening Imaging System}

Figure 1(a) shows a schematic representation of the imaging system constructed for and employed in this investigation, while Fig. 1(b) depicts the system within the surgical suite. The instrument is a hybrid PA/US system, dedicated to BCS imaging applications. It is designed to provide three-dimensional (3-D) PA images with coregistered 3-D US images of surgical samples. The diagram shows a water tank used for acoustic coupling between the sample and PA/US transducer arrays. The linear US array (L14-5/38, Ultrasonix, British Columbia, Canada) provided a $38-\mathrm{mm}$-wide field of view and was operated at a frequency of $6.6 \mathrm{MHz}$. The PA array consisted of 24 disk-shaped PVDF transducers. Each transducer measures $15 \mathrm{~mm}$ in diameter and features a $500-\mathrm{kHz}$ center frequency along with a $120 \%$ fractional bandwidth. The $30-\mathrm{cm}$ diameter PA array was mounted on the effector of a robot (Epson, Japan), as depicted in Fig. 1(c). To perform imaging, a breast tissue sample was positioned in the tank between the robotically guided fused optical fiber and the PA/US arrays. This was accomplished using a custom-designed lumpectomy specimen holder, as seen in Fig. 1(e). The $\sim 30 \mathrm{~mJ}$ pulsed output of the tunable (680 to $950 \mathrm{~nm}$ ) laser (Phocus InLine, Opotek Inc., California) was injected into the fiber and synchronized with the customdesigned 24-channel, 50-MHz data acquisition system (DAQ), so that the laser-induced signals originating from the sample were recorded. The PA/US arrays and fused fiber bundle assembly was then moved to a new scan position using the high-precision four-axis robot, which was controlled using the robot PC. The overall scan was coordinated using a PC with LabVIEW software (National Instruments, Austin, Texas), which controlled the synchronization of the DAQ, robot PC, and laser. The 3-D US scan was carried out using a robot-synchronized clinical US scanner (Sonix Touch, Ultrasonix, British Columbia, Canada).

\subsection{Photoacoustic Array, Calibration, Illumination, and Scan Synergy}

The custom-designed and built 30-cm diameter PA transducer array consisted of 24 disk-shaped PVDF elements measuring $15 \mathrm{~mm}$ in diameter each. The elements were positioned and secured in an arc-shaped aluminum housing [Fig. 1(d)] such that the focus of the array was concentric with respect to the center of curvature. This arrangement, along with the $\sim 15$ - deg angular acceptance of each transducer element, measured at $-6 \mathrm{~dB}$, allowed for an effective isotropic imaging subvolume of $3 \mathrm{~cm} \times 3 \mathrm{~cm} \times 3 \mathrm{~cm}$. Near-complete angular coverage of the imaging subvolume was achieved by rotation of the arc array through 10 steps of $18 \mathrm{deg}$ each. This resulted in 240 projections per subvolume and mimicked the performance of a staring and sparse 240-channel hemispherical array. The spatial locations of the transducers, with respect to the $3 \mathrm{~cm}^{3}$ imaging subvolume, were determined experimentally via robotic calibration scan, developed previously and detailed elsewhere. ${ }^{32-35}$ 
(a)

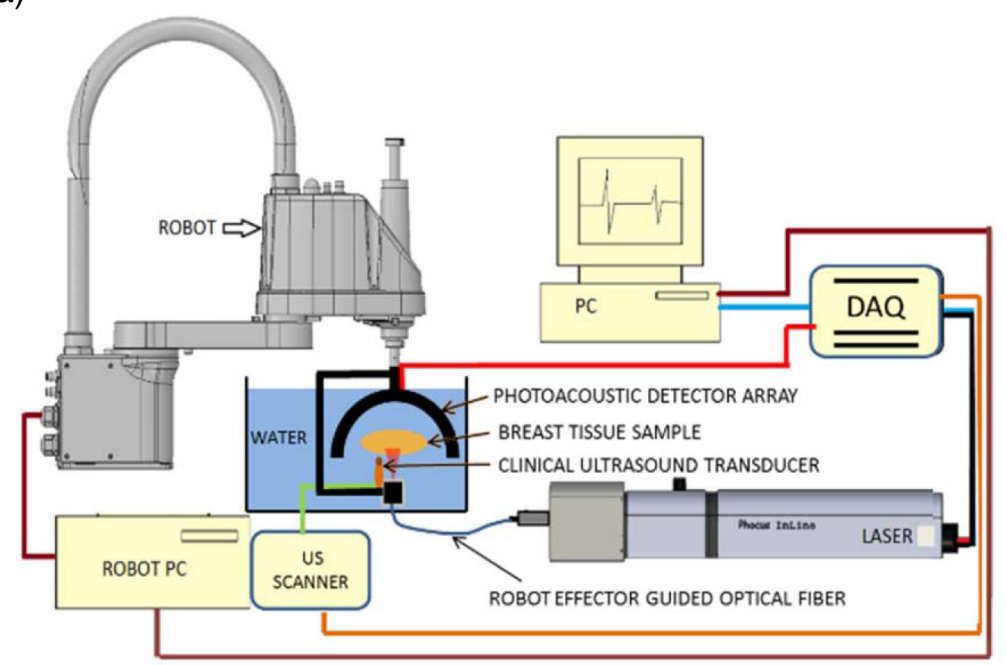

(b)

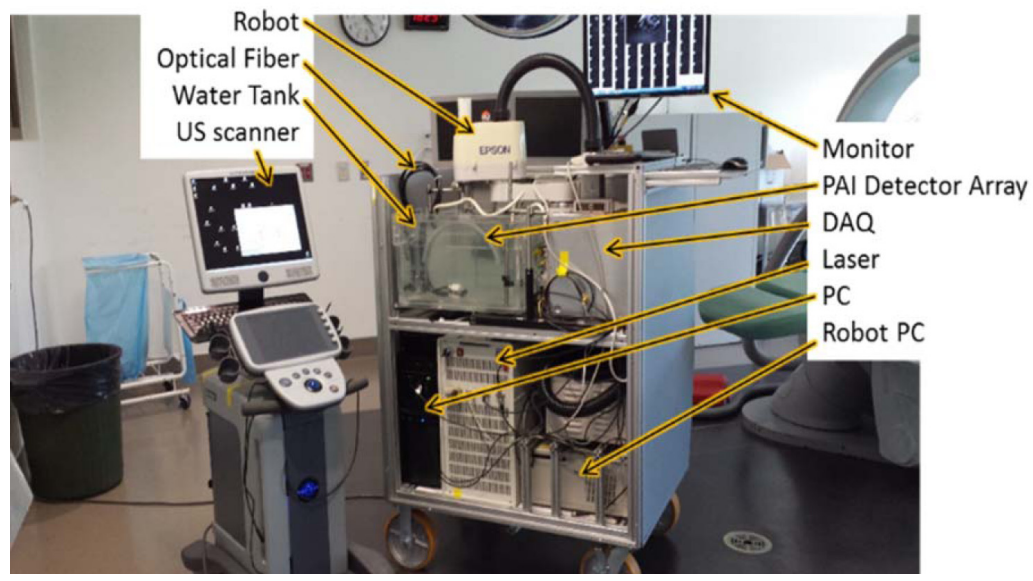

(c)

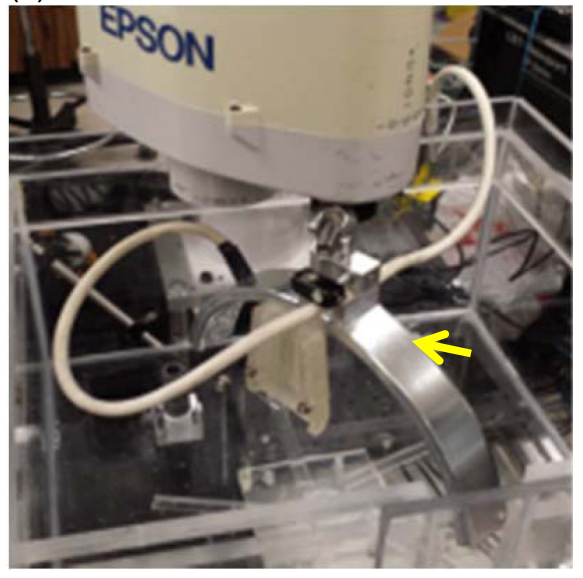

(d)

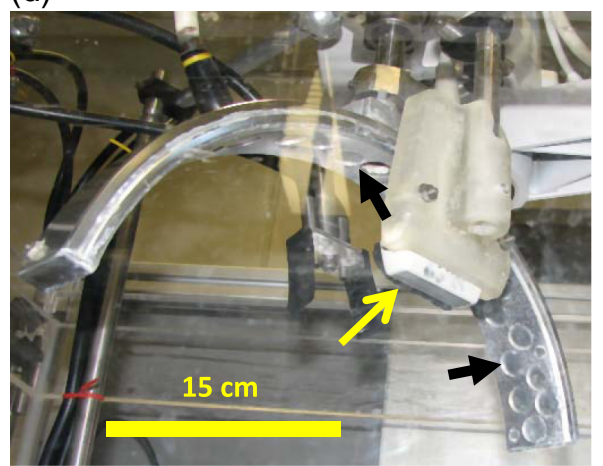

(e)

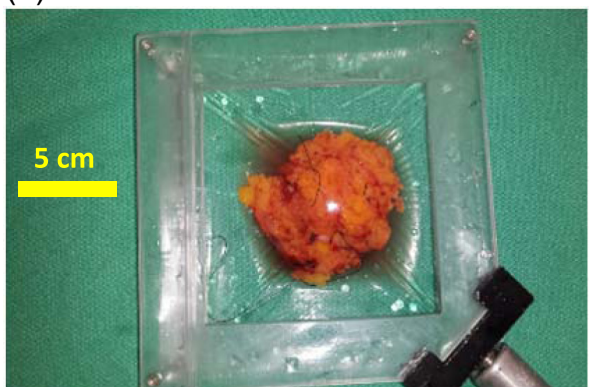

Fig. 1 (a) Schematic representation of the major components of the iPAS system for intraoperative lumpectomy margin evaluation during BCS. (b) A labeled photograph of the intraoperative system within surgical suite, consisting of US system on the left and iPAS system on the right. The front panel of the iPAS system is removed to facilitate convenient viewing of internal components. (c) A close-up photograph of the arc-shaped 24-channel PA transducer array (arrow) mounted on the effector of the Epson SCARA robot. (d) The PA array from below, along with scale bar indicating dimensions and the location of the conventional 6.6-MHz linear US transducer array (yellow arrow). (d) The positioning of the individual transducer elements on the array chassis (black arrows). (e) The custom-built lumpectomy specimen holder which contains a freshly excised compressively restrained lumpectomy specimen.

Importantly, this approach eliminated the dependence of image reconstruction accuracy on array fabrication precision.

To complement the transducer-derived $3 \mathrm{~cm} \times 3 \mathrm{~cm} \times 3 \mathrm{~cm}$ imaging subvolume, a high-power fused-input optical fiber bundle (Lumen Dynamics Group Inc., Mississauga, Ontario, Canada) was positioned $6 \mathrm{~cm}$ below the imaging volume [Fig. 1(a)]. The minimum beam diameter intersecting the volume was also $3 \mathrm{~cm}$. The resulting maximum tissue surface fluence was $\sim 4 \mathrm{~mJ} / \mathrm{cm}^{2}$, which was well below the damage threshold and well below the maximum permissible exposure for in vivo applications. ${ }^{36}$ Furthermore, by illuminating on one side of the specimen while detecting laser-induced PA signals on the opposite side, a transmission imaging mode was implemented. In this mode, the strongest signals originating at the tissue surface were also originating at the largest distance from the transducers, and consequently, they were last to be recorded. As a result, the large signals were not permitted to interact with the detector array until the deeply sourced, weak signals, were recorded. This approach preserved the full dynamic range of the detection electronics and prevented backscattered US "clutter" signals from interfering with direct time-of-flight PA signal measurements. In this way, reflection artifacts commonly associated with deep tissue reflection mode PA imaging were addressed. ${ }^{29}$

To facilitate scans of specimens up to $11 \mathrm{~cm}$ in diameter, the total imaging volume was expanded by horizontal translation of 
the PA array and fiber bundle assembly along with the $3 \mathrm{~cm}^{3}$ imaging subvolume. This was implemented along a square $x-y$ coordinate grid of 100 horizontal scan positions centered $9 \mathrm{~mm}$ apart. Consequently, the imaging scan amounted to 24,000 projections per total imaging volume (i.e., 240 /subvolume $\times 100$ subvolumes). The 24 -channel iPAS system combined with a $20-\mathrm{Hz}$ laser was capable of scanning this total volume of $11 \mathrm{~cm} \times 11 \mathrm{~cm} \times 3 \mathrm{~cm}(L \times W \times H)$ in under 6 min. The iPAS scan was followed by a robotically guided 6.6-MHz US scan, which took $\sim 90 \mathrm{~s}$ and examined the equivalent volume. This volume capacity encompassed the vast majority of lumpectomy specimens, as was indicated by the collaborating surgeon. However, due to a reduction in the iPAS system's signal-to-noise-ratio (SNR), experienced beyond 2 to $3 \mathrm{~cm}$ of imaging depth, the imaging protocol was to invert or flip the specimen and repeat the scan for the opposite side. Nevertheless, the dual-side scan imaging protocol was only implemented when intraoperative time constrains permitted.

Owing to the 20-min time constraint imposed by the intraoperative nature of the imaging protocol, only two wavelengths were able to be utilized in most cases. The expected dominant chromophore distribution inside lumpectomies, namely adipose tissue and deoxyhemoglobin, motivated the use of 930- and 690-nm wavelengths, respectively.

\subsection{Image Reconstruction, Image Stitching, Coregistration and Resolution}

To generate subvolume images using the acoustic time-of-flight measurements performed by the iPAS system, the recorded pressure signals were first passed through a Butterworth bandpass filter (100 to $3 \mathrm{KHz}$ ). The universal backprojection (BP) algorithm was then implemented. ${ }^{37}$ The BP method preserved the rich frequency content of broadband PA signals, necessary for generating boundary-enhanced images. The full imaging volume was composed of 100 individual subvolumes measuring $3 \mathrm{~cm}^{3}$ each and centered $9 \mathrm{~mm}$ apart. Consequently, stitching these subvolumes together resulted in significant overlap or heterogeneous spatial oversampling. This effect, combined with the laser output variability from shot to shot, manifested in the final composite image as a noticeable grid pattern. To compensate for the resulting uneven bulk-voxel intensity between neighboring subvolumes, image stitching was carried out in the gradient domain using the Haar wavelet twodimensional integration method. ${ }^{38} \mathrm{~A}$ disk-shaped $1 \%$ agar gel phantom with radially directed graphite spokes was prepared to evaluate the performance of gradient domain stitching versus spatial domain stitching. The graphite concentration in the spokes was $1 \mathrm{~g} / \mathrm{L}$. After the phantom was fully cured it was secured in the iPAS water tank and scanned at a wavelength of $690 \mathrm{~nm}$.

Coregistration of the iPAS and US images was performed using Horn's absolute orientation method. ${ }^{39}$ The method called for a minimum of three known coordinates in each of the two (PA and US) Cartesian coordinate systems. An agar gel phantom with four spherical graphite-containing inclusions was prepared in order to calibrate the two systems. The phantom was scanned using the iPAS system at a wavelength of $690 \mathrm{~nm}$, and using the US system at 6.6 MHz. The inclusions were clearly visualized on both the US and iPAS images and the center coordinates of the spheres were selected for coregistration. In terms of precision and accuracy of the calibration, two factors reduced the achievable agreement between the two imaging modalities.
First, the limited iPAS resolution implied that considerable uncertainty was present when selecting the center coordinates of the coregistration targets. Therefore, the accuracy of the coregistration is dependent on the resolution and hence limited. Moreover, the coregistration precision was impacted by unwanted water turbulence in the tank. The turbulence was caused by the moving transducer array and produced millimeter-level deviation of the specimen position. These limiting factors should be kept in mind when making detailed comparisons of the results.

An imaging target consisting of a black surgical suture was selected to determine the iPAS system resolution. Full width at half maximum (FWHM) analysis of the line profile of the suture target was carried out to estimate the effective resolution value. The BP, image stitching, and coregistration were all performed in MATLAB.

\subsection{Lumpectomy Specimen Collection and Preparation}

Patients admitted to the regional breast care center for screening mammography or symptomatic breasts were evaluated by an experienced radiologist. In cases of BI-RADS 4 or 5 classification, the patients were referred for a stereotactic biopsy procedure. Those diagnosed with malignancy (BI-RADS 6) and scheduled to undergo BCS were asked to participate in the study. Patient inclusion criteria followed the outline in the Patient Inclusion/Exclusion Diagram found in Supplementary Material. All studies were carried out with informed and signed consent as well as the approval of the institutional review board (IRB) of The University of Western Ontario (UWO Research Ethics Board \# 105467; LHSC Tissue Archive \# 888; Lawson Approval \#R-14-311).

The intraoperative imaging protocol permitted the surgical standard of care to be unaffected by the iPAS and US imaging scans. The freshly excised lumpectomy specimens were placed inside a customized saline-filled Ziploc ${ }^{\circledR}$ bag and handed to the iPAS specialist for scanning. In the meantime, the patient was sutured and prepared for surgical suite discharge. Prior to discharge the specimen was handed back to the surgeon, who then passed it on to pathology. Following specimen fixation, a pathological examination was completed and a breast invasive carcinoma synoptic report was generated, according to AJCC/UICC TNM 7th edition, CAP Version 3.1.0.0 (June 2012). The work was completed by, or under supervision of, a senior pathologist. The synoptic report was used to determine the tumor type, grade, extent, and position within the specimen. This preliminary report was based on the first 52 out of 200 patients approved by our IRB for iPAS investigation. In this proof-ofconcept pilot study, we have highlighted seven unique cases.

\section{Results}

\subsection{Image Reconstruction, Stitching, and System Resolution}

The result of image reconstruction and stitching in the gradient domain, as compared to the spatial domain, can be appreciated in Fig. 2. The figure shows outcome of scanning the spoked agar/graphite gel phantom followed by BP reconstruction and subvolume stitching in the spatial domain [Fig. 2(a)], and in the gradient domain [Fig. 2(b)]. The selected $z$-slices are separated by $1 \mathrm{~mm}$ and depict the graphite spoke inclusions as 


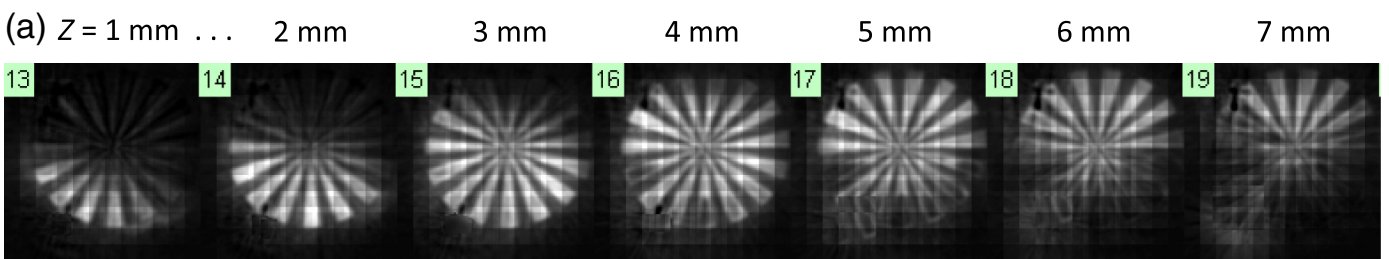

(b)

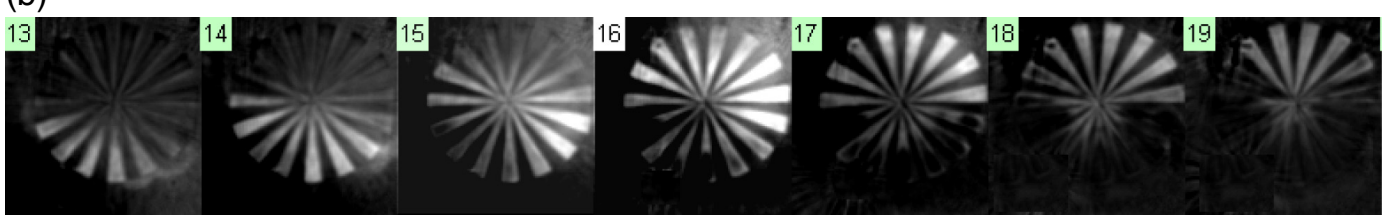

(c) Spatial domain stitching

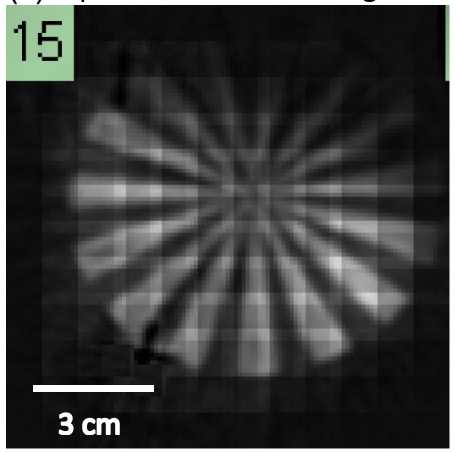

(d) Gradient domain stitching

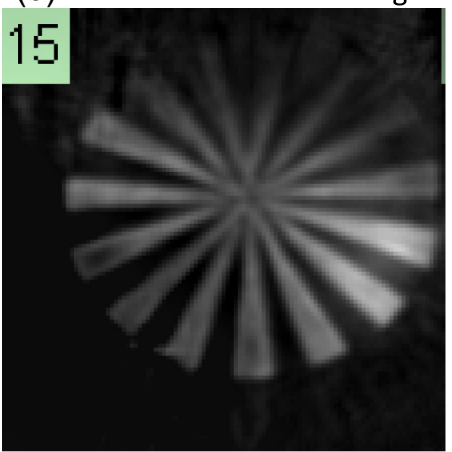

(e)

Photograph

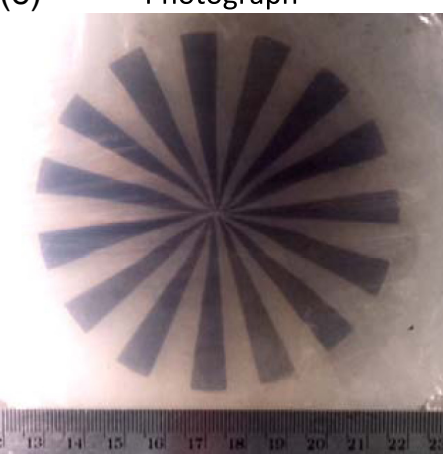

Fig. 2 (a) Selected 690-nm iPAS $z$-slices showing a graphite/agar gel phantom reconstructed using spatial domain stitching, resulting in unwanted scan grid artifacts in the final composite image. (b) The same scan data but with stitching implemented in the gradient domain, resulting in complete or near complete elimination of the grid artifacts. The larger images in (c) and (d) allow for an easier appreciation of the result, while the photograph in (e) provides an effective gold standard reference.

hyperintense on the iPAS scan. On the other hand, the photograph in Fig. 2(e) shows the same graphite inclusions as dark gray. The effect of uneven illumination and heterogeneous spatial sampling between the stitched subvolumes, manifested in the final composite image as a grid pattern, which is easier to see in the zoomed-in slice in Fig. 2(c). Intriguingly, the scan-grid artifacts appear to be effectively eliminated by implementing stitching in the gradient domain, as shown in Fig. 2(d).
Equally important, however, is the apparent lack of any significant side effects on the final image results.

Figure 3 shows the result of FWHM analysis of the line profile of a surgical suture. Figure 3(a) shows a photograph of a freshly excised lumpectomy specimen oriented with respect to the surgical cavity using $\sim 50-\mu \mathrm{m}$ diameter black surgical sutures (white arrows), which have been attached to the specimen by the surgeon. The appearance of the black sutures, seen (a)

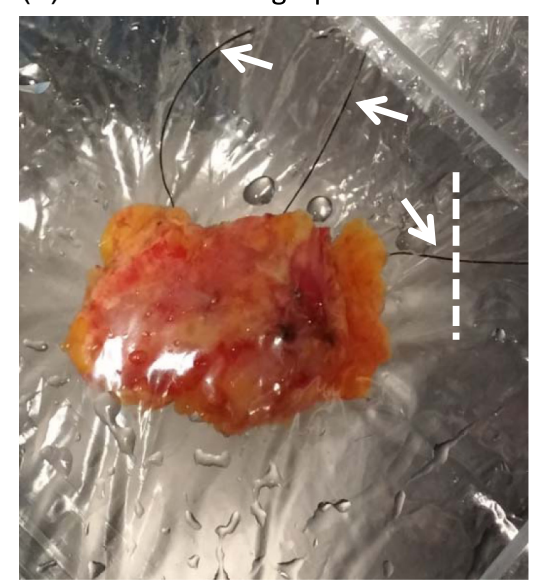

(b)

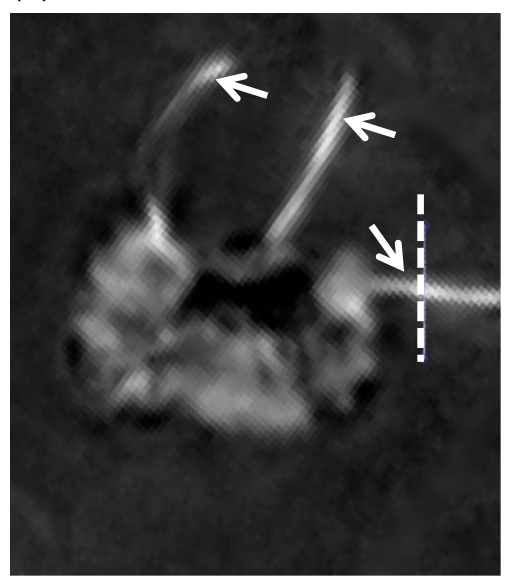

(c) Line profile plot

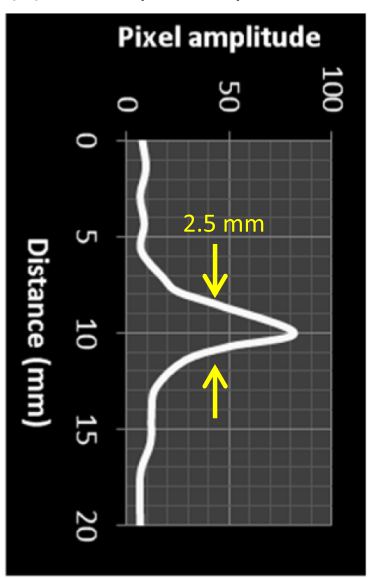

Fig. 3 The iPAS system resolution evaluation via line profile analysis of surgical suture image. The black sutures, indicated by white arrows in the photograph of panel (a), are clearly visualized on the 930-nm iPAS scan result of (b). The suture, indicated by an orthogonal $20-\mathrm{mm}$ dashed line in the photo and iPAS image, is used to estimate the in-plane resolution of the imaging system. FWHM analysis indicates a resolution of $\sim 2.5 \mathrm{~mm}$. 
as hyperintense on the $930 \mathrm{~nm}$ iPAS scan, can be appreciated on the selected iPAS slice in Fig. 3(b). The dashed line in the photograph, and on the iPAS slice, indicates the region that generated the line profile plot seen in Fig. 3(c). Based on the FWHM of the pixel amplitudes, plotted as a function of distance along the line, a resolution of $2.5 \mathrm{~mm}$ was obtained.

\subsection{Intraoperative Photoacoustic Screening Imaging of Freshly Excised Lumpectomies}

\subsubsection{Case study 1. Invasive ductal carcinoma excision with radioactive seed localization}

A 69-year-old patient presented with an architectural distortion on screening mammography and subsequently underwent an US exam of the whole right breast. The exam revealed an $\sim 16-\mathrm{mm}$ diameter hypoechoic mass which demonstrated irregular margins and posterior acoustic shadowing. Based on the mammography and US results the lesion was classified as BI-RADS 5. Consequently, an US-guided biopsy was carried out which resulted in an IDC diagnosis (BI-RADS 6). The patient underwent BCS with radioactive seed localization. Postoperative pathology discovered an 18-mm diameter, grade II, IDC lesion with the radioactive localization seed embedded within. The lesion and localization seed (arrow) can be appreciated in Fig. 4(e), which depicts an intraoperative transmission x-ray image of the specimen. Figures 4(a)-4(d) show representative $z$-slice image stacks of the freshly excised breast lumpectomy specimen at the indicated depths. Figures 4(a) and 4(b) were acquired using the iPAS scanner at 690 and $800 \mathrm{~nm}$, respectively. Little correlation of internal structure was found when comparing the 690 and $800 \mathrm{~nm}$ hemoglobin-enhanced iPAS images with the US results of Fig. 4(d). On the other hand,

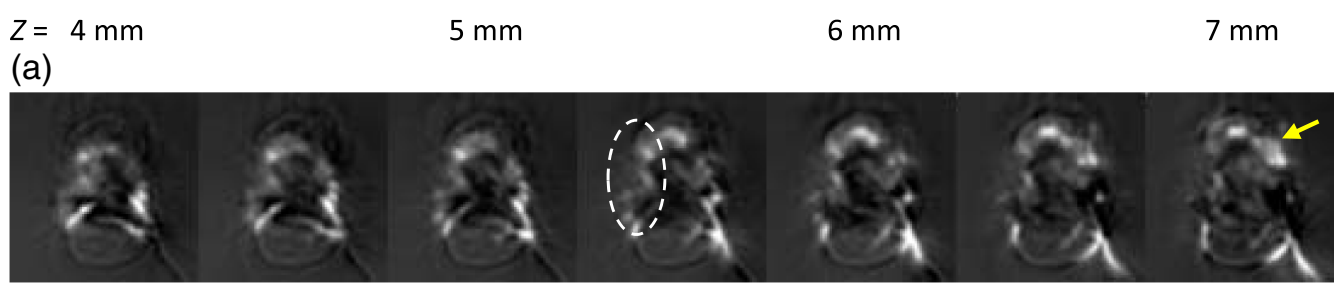

(b)

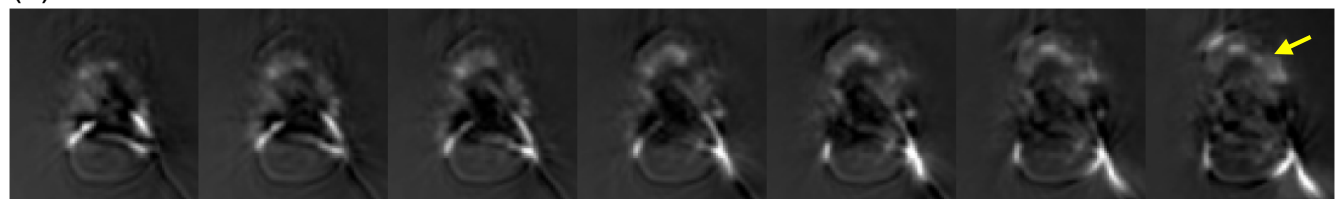

(c)

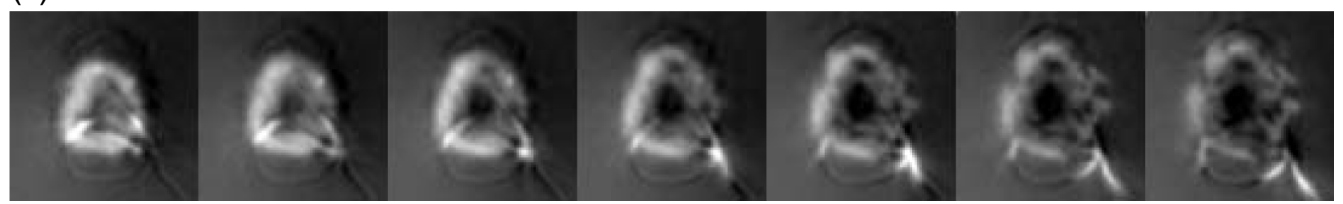

(d)

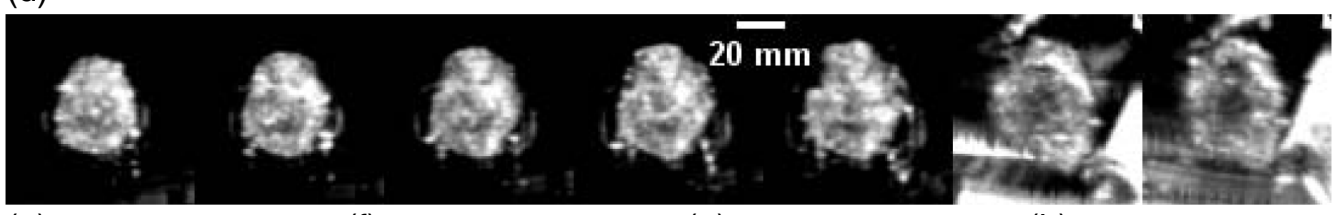

(e) X-Ray

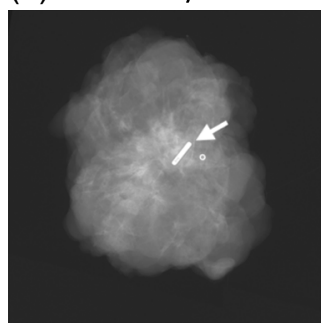

(f) iPAS

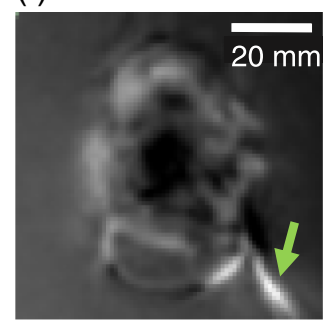

(g) US

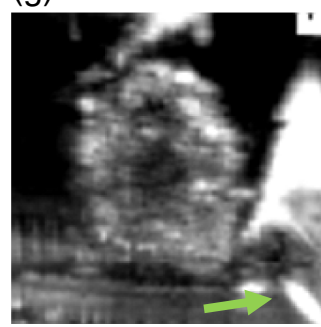

(h) Photograph

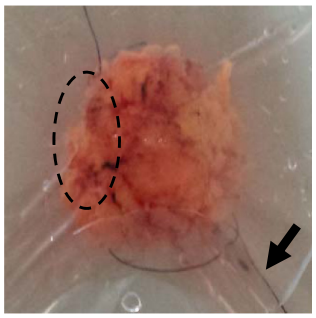

Fig. 4 Image stacks of lumpectomy specimen containing an 18-mm diameter, grade II IDC, acquired using the iPAS system at the indicated $Z$-depths below the illuminated surface. (a) and (b) iPAS results for 690 - and $800-\mathrm{nm}$ scans, respectively, illustrating the small difference between targeting deoxyhemoglobin $(690 \mathrm{~nm})$ and total hemoglobin $(800 \mathrm{~nm})$. The increased intensity of the $690-\mathrm{nm}$ scan results, compared to $800 \mathrm{~nm}$ (yellow arrows), likely indicates the presence of mostly deoxygenated blood in the excised specimen. Nonetheless, both wavelengths fail to unambiguously differentiate the malignancy region, best indicated in the near 7-mm-deep slice of the 6.6-MHz US scan shown in (d) and (g), as well as in the x-ray image found in (e). On the other hand, the iPAS slices in (c) and (f), acquired using lipid-weighted $930-\mathrm{nm}$ scans, clearly show a $20-\mathrm{mm}$ diameter centrally located hypointense area corresponding to the $\mathrm{x}$-ray and US findings. 
the 930-nm lipid-enhanced iPAS images in Fig. 4(c) clearly show a dark central mass corresponding to the hypoechoic region found in the US images of Fig. 4(d). This is easier to confirm in the zoomed-in images of Figs. 4(f) and 4(g), where the US and the 930-nm iPAS slices show a hypointense region measuring $\sim 20 \mathrm{~mm}$ in diameter. Furthermore, these findings were found concordant with the intraoperative x-ray image in Fig. 4(e) and the pathology report, as both confirmed the central location of the IDC tumor within the lumpectomy specimen. For reference, Fig. 4(h) shows a color photograph of the specimen (not coregistered), taken immediately prior to the iPAS scans.

\subsubsection{Case study 2. Failed lumpectomy surgery: Margin positive for ductal carcinoma in situ}

Figure 5 summarizes the results of imaging a lumpectomy specimen which was confirmed by pathology to contain a positive margin and resulted in a repeat surgery. A 63-year-old patient was diagnosed with a left-sided IDC via US-guided biopsy (BIRADS 6) and underwent BCS. Owing to palpable nature of the lesion, excision was performed without seed- or wire-guided localization and produced the specimen shown in photograph of Fig. 5(e). Figures 5(a) and 5(c) depict selected 930-nm
iPAS slices at the indicated depths and orientation, with superior at the top and anterior to the right (yellow arrows). Inspection of Fig. 5(a) demonstrates a large hypointense mass located centrally through to a depth of about $10 \mathrm{~mm}$. However, at a depth of $11 \mathrm{~mm}$, an apparent extension of the mass reaches toward the superior margin. This is best visualized in Fig. 5(c) where the affected margin is indicated (white arrow). Interestingly, the coregistered US results of Figs. 5(b) and 5(d) also indicate concordant hypointensities; nonetheless, the superior margin there appears intact. Postoperative pathologic examination of the specimen revealed a grade II IDC lesion measuring $40 \mathrm{~mm}$ in diameter and reaching within $2.5 \mathrm{~mm}$ of the superior margin. Moreover, pathology further discovered an extension of the DCIS tumor which infiltrated the superior margin, resulting in a failed excision. To minimize the risk of recurrence, the patient underwent a second procedure approximately 1 month later.

\subsubsection{Case study 3. Hypervascularized invasive ductal carcinoma}

An 88-year-old patient was admitted at the breast care center due to palpable lump in the right breast and subsequently underwent BCS. Figure 6(a) shows the results of an US scan while

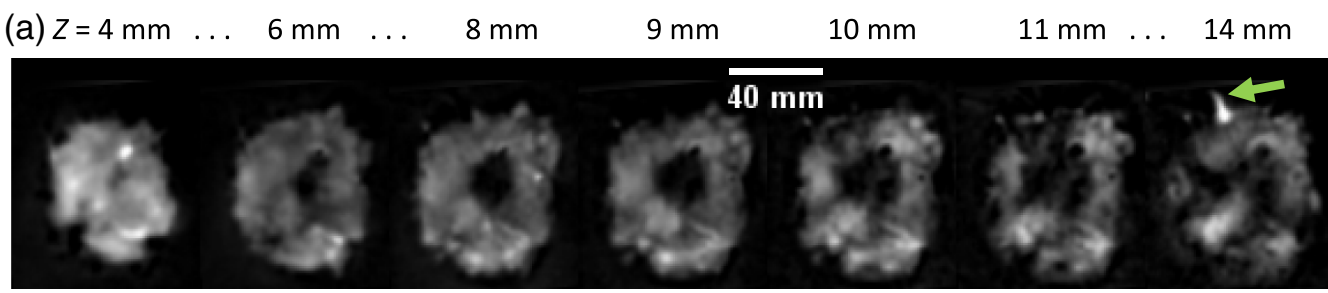

(b)

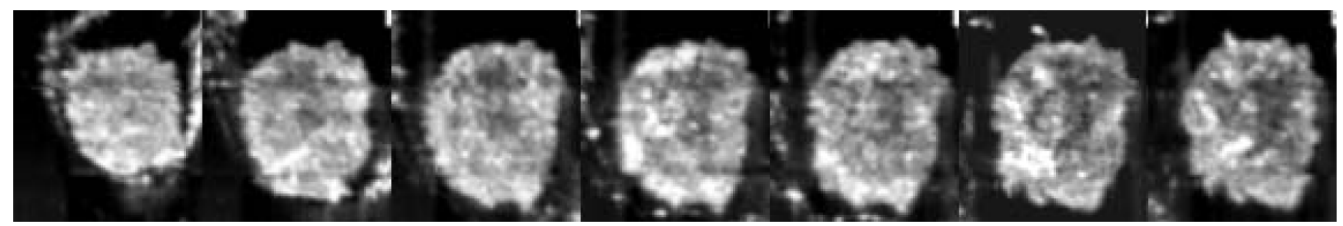

(c) $Z=11 \mathrm{~mm}$ iPAS

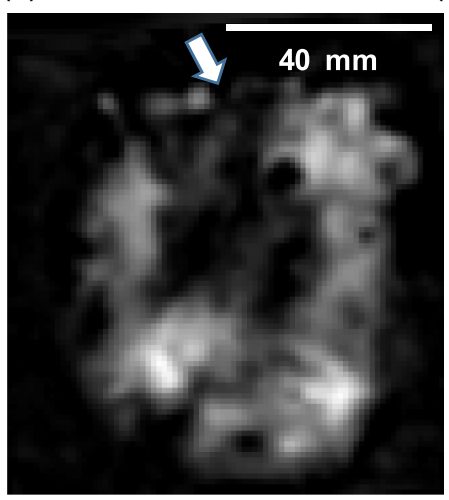

(d) $\quad Z=11 \mathrm{~mm}$ US

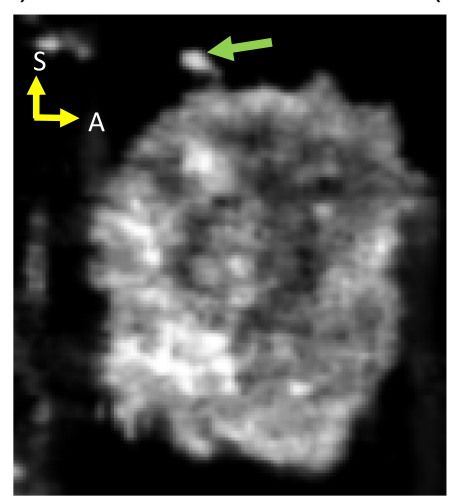

(e)

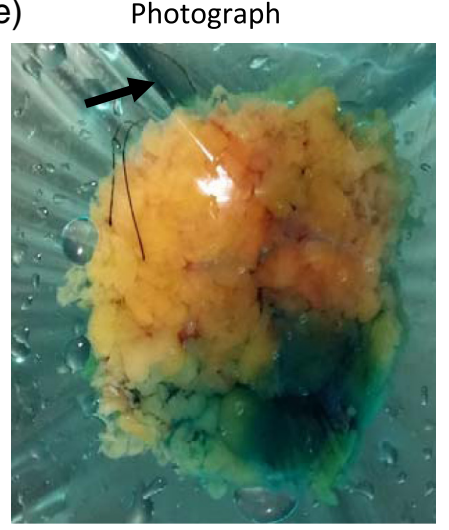

Fig. 5 (a) The 930-nm iPAS slices corresponding to the indicated depth below the illumination surface, showing a grade II IDC along with a grade II DCIS-containing specimen, found by postoperative pathology to have a positive margin. (b) Coregistered slices acquired using a 6.6-MHz conventional US scanner. (c) and (d) A larger view of the slice near $Z=11 \mathrm{~mm}$ for iPAS and US, respectively. The iPAS slice in (c) clearly shows intrusion of the specimen edge by the hypointense extension (white arrow). For reference, a photograph is included in (e), which, along with the 14-mm iPAS and US slices, shows the superior orientation suture (black and green arrows). The sutures facilitate the orientation of the specimen with superior $(\mathrm{S})$ at the top and anterior $(\mathrm{A})$ to the right (yellow arrows). 
(a)

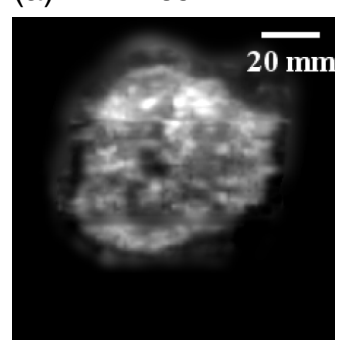

(b) iPAS $930 \mathrm{~nm}$

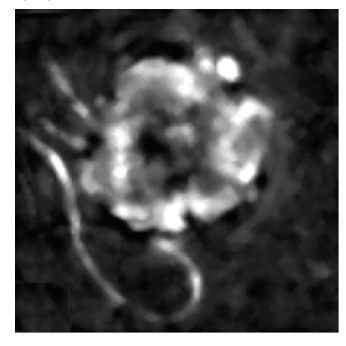

(c) iPAS $690 \mathrm{~nm}$

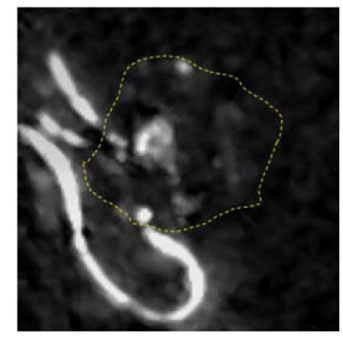

(d) Photograph

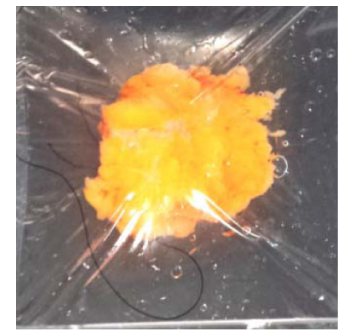

Fig. 6 The iPAS assessment of lumpectomy specimen belonging to an 88-year-old patient confirmed by pathology to contain a 15-mm grade I IDC along with grade II DCIS. Interestingly, in this case, the hypointense tumor areas found in the US and $930 \mathrm{~nm}$ iPAS images of (a) and (b) correspond well to the hyperintense area in the $690 \mathrm{~nm}$ iPAS image of (c). This is likely caused by the simultaneous presence of increased vascularity along with decreased lipid content of the tumor region. For easier comparison the dashed yellow line in the 690-nm iPAS image indicates the outer perimeter of the specimen which is difficult to see due to the low hemoglobin content of the healthy margin. For reference, a photograph is included in (d).

Figs. 6(b) and 6(c) depict images from an iPAS scan performed at lipid-weighted $930 \mathrm{~nm}$ and hemoglobin-weighted 690-nm wavelengths, respectively. The figure shows an excised lumpectomy confirmed by pathology to contain a $15-\mathrm{mm}$ diameter grade I IDC along with grade II DCIS. In this example there is a good agreement between the hypointense areas in the US and 930-nm iPAS images, compared to the hyperintense area in the 690-nm iPAS image. This is likely caused by the simultaneous presence of increased vascularity along with decreased lipid content of the tumor region. However, this amount of agreement is rare as hemoglobin-enhanced features on iPAS, if any, were more typically found near the periphery of tumors or in seemingly nonrelated areas, reducing the specificity of the results. Nevertheless, this case indicates that, at least from a technical perspective, the iPAS system is capable of visualizing hypervascularization associated with tumor regions.

In addition to visualization of malignant tissue within the lumpectomy specimen, two imaging features, exemplified by the results shown in Figs. 4-6, should be pointed out. First, notice the bright streaks especially apparent near the bottom and on the right of the US slices, near the 7-mm depth in Figs. 4(d) and 4(g). These streaks are caused by US visualization of the $\sim 40-\mu$ m-thick polyethylene Ziploc ${ }^{\circledR}$ bag, which is used to restrain the lumpectomy specimen. The streaks typically became apparent near the midplane of the specimen where the thin bag material obliquely intersected the imaging plane. Because of the low near-infrared absorption coefficient of the optically clear Ziploc ${ }^{\circledR}$ bag material, it is not visualized in the iPAS images. Second, inspection of the photographs in Figs. 4 and 5 reveals the presence of black surgical sutures (black arrows), which are used by the surgeon to mark the orientation of the specimen with respect to the patient's body. These are also visualized in the iPAS and US images (green arrows).

Figure 7 depicts selected slices demonstrating iPAS visualization of four types of lesions frequently encountered in BCS. The figure contains intraoperative $\mathrm{x}$-ray images as well as iPAS and US results for the selected specimens. The shown slides have been cooriented to allow a comparison of feature locations. Briefly, Fig. 7(a) is an example of a BCS specimen containing a biopsy-proven 17-mm-diameter grade I IDC tumor. Results of inspecting the US image seem to be inconclusive as just a few contrasting features are present (arrows) in the area marked by the radioactive localization seed and architectural distortion, seen in the x-ray image (dashed circle). On the other hand, the 930-nm iPAS image illustrates a specimen with an $~ 20-\mathrm{mm}$ diameter hypointense mass surrounded by a hyperintense cuff produced by the high lipid content of the healthy margin enclosing the tumor. The last column shows the corresponding photograph, which, along with the x-ray, is not coregistered. Figure 7(b) shows similar results; however, in this case the lesion is composed of a combination of an 8-mm diameter grade I IDC and a grade II DCIS tumor. Again, in comparison to the x-ray results, the iPAS image appears to indicate more concordant contrasting features while US fails to unambiguously define the extent of the lesion. The specimen shown in Fig. 7(c) is described by the pathologist as containing two apparently separate foci of grade I IDC tumor invasion, measuring 16 and $15 \mathrm{~mm}$ in diameter each, contiguously located with highgrade (III) DCIS. Interestingly, the 930-nm iPAS image in this case agrees with the $\mathrm{x}$-ray and also US, as all show extensive contrasting features extending through much of the specimen. Finally, Fig. 7(d) depicts a specimen containing a pure DCIS lesion, measured on pathology to be at least $18 \mathrm{~mm}$ in extent. The lesion is excised using wire-guided localization, appreciated in the x-ray and photograph, which show the hooked localization wires inserted in the lesion (arrows). Consistent with the previous performance, the iPAS image shows hypointense features whose location coincides with the apparent lesion location in the X-ray image, marked by the hooked wire ends. Again, the US results are inconclusive as there are few definite contrasting areas in the image.

\section{Discussion}

\subsection{Lipid versus Hemoglobin in Ex Vivo Applications}

A survey of PA imaging literature suggested that the primary contrast of tumors was provided by elevated hemoglobin concentration associated with cancer-induced angiogenesis. ${ }^{22,25,40-42}$ Consequently, it was expected that 690 -nm imaging would show increased signal intensity originating from periphery of tumor regions. However, contrary to this hypothesis, this investigation found evidence that 690 -nm deoxyhemoglobin-weighted images generally showed little or no correlation with hypoechoic US regions inside ex vivo specimens. This was the case for most of the specimens encountered in this study and has 
(a) $\quad$-ray

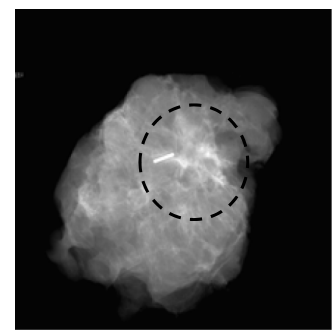

(b)

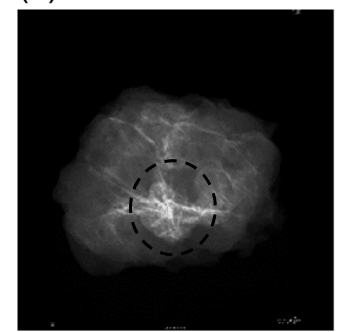

(c)

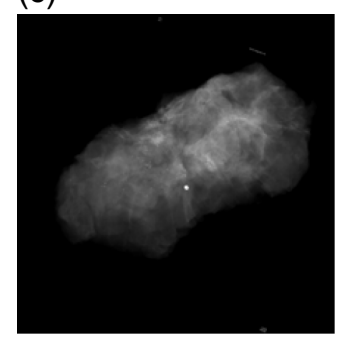

(d)

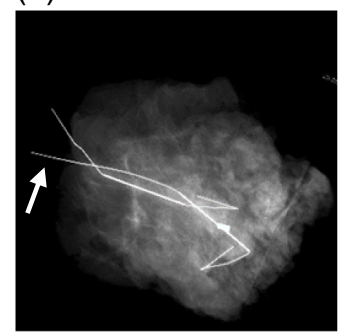

iPAS
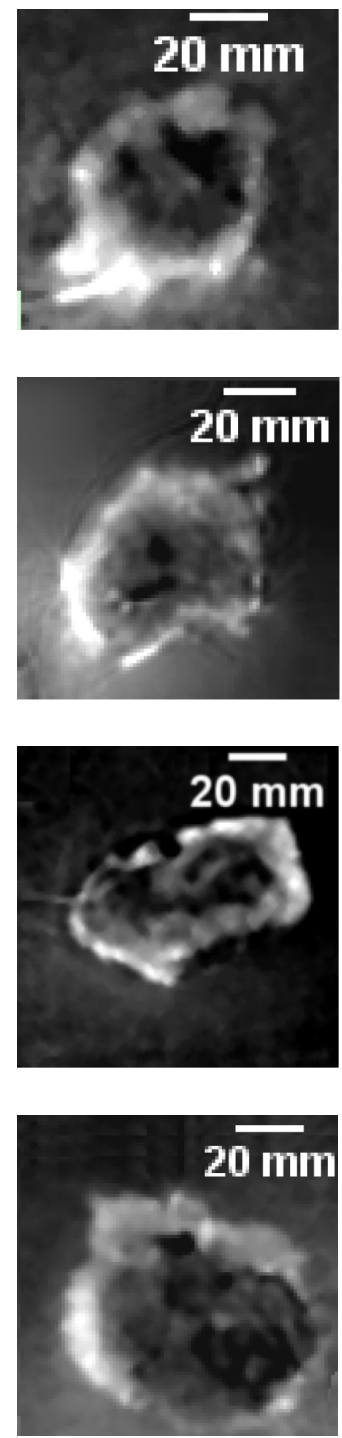

US
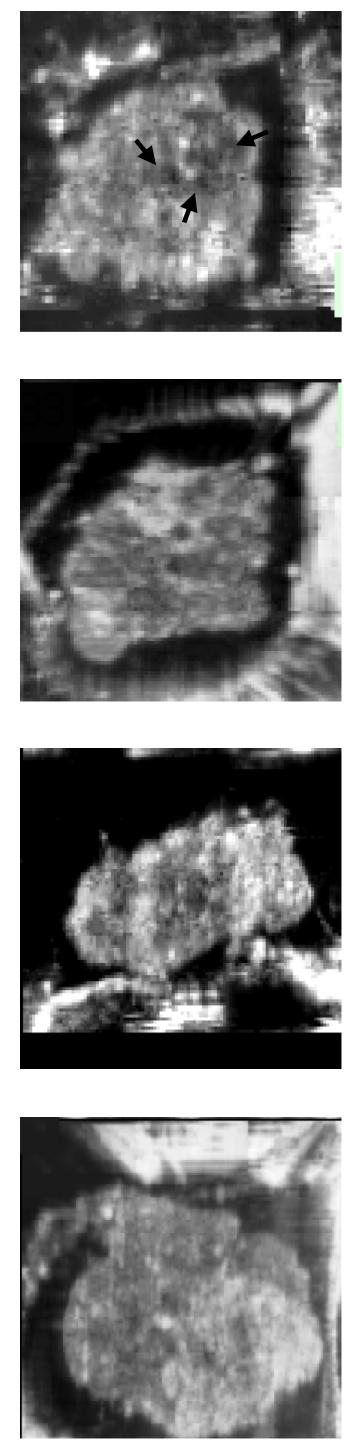

Photograph
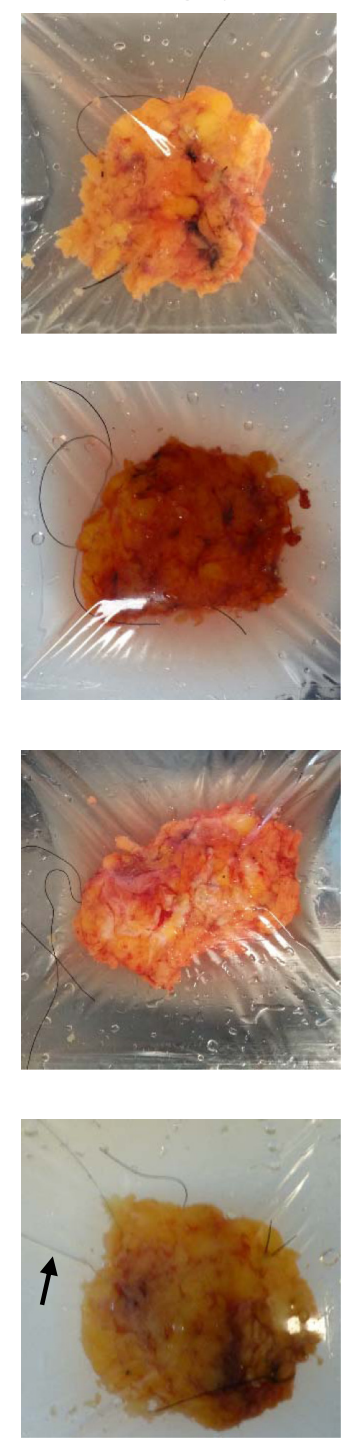

Fig. 7 Montage with slides of commonly encountered BCS lesions, acquired using the iPAS lumpectomy evaluation system. Note that these results may not be representative of all cases. The first column shows intraoperative transmission $x$-ray images. The second column depicts the corresponding 930-nm iPAS slices, which are coregistered to the US slices shown in the third column. Finally, the last column shows specimen photographs, which, along with the x-ray images, are not coregistered. The specimen in (a) contained a pathologically confirmed 17-mm IDC with concordant findings on IPAS and $x$-ray (dashed circle), and to a lesser degree, US. (b) A specimen which contained a lesion composed of an 8-mm IDC with an extension of DCIS. (c) A lumpectomy specimen found by pathology to contain two adjacent foci of IDC, measuring $16 \mathrm{~mm}$ and $15 \mathrm{~mm}$ each, along with high-grade DCIS. Last, the lumpectomy found in (d) is confirmed to be a pure DCIS lesion measuring at least $18 \mathrm{~mm}$ in diameter and is removed using wire-guided excision. The hooked localization wires can be appreciated in the photograph and on the $x$-ray image (arrows). The iPAS results appear to be in agreement as they show hypointense areas corresponding to the lesion location in the $\mathrm{x}$-ray.

been exemplified in Fig. 4. On the other hand, Fig. 6 has demonstrated that, at least in certain cases, the iPAS system was capable of visualizing hypervascularity in tumors. Therefore, the extendibility of documented in vivo results to ex vivo applications must be questioned. The extendibility hypothesis rested upon two assumptions. First, it was assumed that a freshly excised tissue specimen will quickly reach low blood oxygenation levels due to a lack of blood supply. This motivated the 690-nm wavelength selection, where deoxyhemoglobin dominates tissue absorption. The validity of this assumption was corroborated by the increased signal intensity, seen in the 690-nm slices compared to the 800-nm slices of Fig. 4 (yellow arrows). Specifically, one can infer that the blood content in the excised tissue was dominated by deoxyhemoglobin since the 800 -nm wavelength was absorbed equally by both deoxy hemoglobin and oxyhemoglobin, while the 690-nm wavelength was primarily absorbed by only deoxyhemoglobin. Indeed, upon closer inspection of the 690-nm iPAS imaging results of Fig. 4, it can be seen that areas with higher blood concentration produced high-intensity features. For example, the area of high 
blood content marked in the photograph of Fig. 4(h) by a dashed oval, corresponded well to the iPAS 690-nm image in Fig. 4(a), near the periphery of the specimen (dashed oval). The second assumption was that the blood distribution inside the specimen would remain relatively unchanged after excision. However, the blood distribution depended on the ability of the vascular network to maintain blood vessel volume, which in turn depended on the blood pressure and cardiac output. Because an excised specimen was no longer connected to the cardiovascular system, it was possible that many of its blood vessels collapsed during surgery, leading to a heterogeneous reduction in blood volume. Therefore, hemoglobin distribution inside excised tissue specimens may not be a good biomarker of malignancy.

Alternatively, low lipid concentration has been well documented by optical methods as being a significant indicator of breast abnormalities. ${ }^{43}$ For example, a recent broadband nearinfrared mammography study of 26 breast cancer patients found significantly altered chromophore concentrations in disease-affected areas of the breast. In particular, the reduced lipid concentration was among the strongest indicators of malignancy. Moreover, the researchers also examined the Nottingham histologic score of each tumor, essentially a measure of the biological aggressiveness of the disease, and compared it to the level of lipid reduction in the associated lesion. Intriguingly, a significant relationship was found, indicating that the most aggressive lesions tended to alter the lipid content the most. Therefore, beyond simply detecting malignant tumors, lipid-weighted breast tumor imaging may also be able to estimate the aggressiveness of the disease. This outcome may prove highly valuable since early detection of particularly aggressive cancers has been one of the most important prognostic factors.

Furthermore, the lipid concentration within breast tissue, including the parenchyma and adipose tissue, is unaffected by surgical excision. Consequently, it is possible that low lipid concentration is a good indicator of tumor location and extent, in excised tissue. Moreover, in addition to excised tissue, lipid may also be an effective biomarker for in-vivo tumor detection. Therefore, researchers with access to high-fidelity PA imaging systems should make an effort to examine this approach, which may improve the specificity of the imaging outcomes. The preliminary results of the study presented here provide motivation for this direction.

Finally, the results outlined in the case study sections provided confidence that PA imaging may have a significant role to play in soft-tissue visualization and assessment, especially as pertains to malignancy visualization. The high concordance of iPAS results with established x-ray and US technology, in terms of tumor location and extent, were encouraging. Furthermore, the agreement between iPAS-derived indications and tumor characteristics derived from the pathology report, such as tumor type and extent, indicated that molecular specificity of optical imaging may give iPAS an edge over existing lumpectomy margin assessment methods. In particular, the positive margin case illustrated a possible opportunity to intervene intraoperatively, potentially sparing the patient a repeat surgery; however, substantially more data must be gathered before conclusions are made.

\subsection{Enabling Intraoperative Surgical Intervention}

Mapping 3-D spatial coordinates of detected positive margins to the surgical cavity where missed malignant tissue needs to be excised is not trivial. Fortunately, the current standard of practice for BCS surgery includes specimen orientation sutures, which are used by the surgeon to mark the lateral (long suture) and superior (short suture) orientation of the specimen with respect to the patient. These can be seen in the specimen photographs. Even more importantly, the high-contrast appearance of black color sutures in the iPAS images promises convenient referencing of the specimen orientation with respect to the surgical cavity, enabling intraoperative intervention in cases of positive margin detection.

As outlined in the introduction, most systems currently being considered for intraoperative margin assessment applications do not provide the surgeon with a full view of the tumor and, at worst, only examine the surface. In some cases, this approach may not meet the needs of the standard of care, but perhaps even more importantly, it may hinder the translation of the technology to the clinic. Complete visualization of the specimen, which is currently a popular intraoperative standard provided by x-ray imaging, allows evaluation of the tumor extent, shape, morphology, and position within the specimen. The information is provided simultaneously, giving the surgeon confidence about the assessment results, and this likely plays a role in enabling effective intervention. The iPAS system is able to deliver this familiar functionality, but with the enhanced sensitivity and specificity of optical spectroscopy.

\subsection{System Resolution, Coregistration and Scan Speed}

The estimated $2.5-\mathrm{mm}$ resolution of the iPAS system is sufficient for most intraoperative applications. The present surgical standard of a minimum 2-mm clear margin is potentially practicable with the current version of the iPAS system. However, the ongoing debate about the minimum margin width necessary for recurrence prevention may establish a new standard of $<2 \mathrm{~mm}$, in the near future. Fortunately, this trend would not render iPAS ineffective because the system resolution can be readily increased by the use of higher-frequency PA transducer arrays.

A resolution of $2.5 \mathrm{~mm}$, combined with millimeter-level specimen position deviation caused by water turbulence, implied that coregistration errors of up to $\sim 4 \mathrm{~mm}$ were possible. Moreover, accurate quantification of total coregistration error was difficult to determine, since it depended on the amount of specimen position deviation which in turn depended on the size and shape of the specific specimen being examined. Therefore, future development of the intraoperative system should include more effective iPAS-compatible specimen immobilization methods and/or an external motion tracking system, which could be used to provide coregistration correction information. These countermeasures should reduce the coregistration error to a level that will not adversely affect the clinical interpretation of the imaging results.

The scan time of 6 min per side meant that scanning both sides required about $12 \mathrm{~min}$, not including sample preparation and inversion. This was substantially longer than just a few seconds needed by current x-ray technology. However, we used a 24-channel PA array and, consequently, the system spent most of its time rotating and translating the array over the imaging volume. An array with 240 elements can be constructed using a straightforward extension of existing technology. This would result in a scan time of $<40 \mathrm{~s}$, on par with current $\mathrm{x}$-ray technology. 


\section{Conclusion}

We have presented preliminary results of the intraoperative PA imaging of breast lumpectomy specimens using an iPAS scanner, clearly showing whole tumors in a true clinical setting. This has been accomplished via construction of a medical-grade carted PA imager. A key aspect of PAT system design is the use of transducer array technology specialized for a specific imaging task. Detecting signals from large tissue specimens requires highly sensitive detectors with frequency bandwidth extending over an ultrabroad range, typically unavailable commercially. Furthermore, accurate reconstruction of PA images requires full-view PA detection geometry. By addressing these common pitfalls of PAT system design we have successfully constructed an intraoperative PAT lumpectomy evaluation system allowing us to pursue practical imaging studies within clinical conditions. We have found that targeting surgical margins during BCS imaging is advantageous over targeting the tumor due to the consistent presence of lipids in healthy breast tissue. This approach shines a light toward soft tissue abnormality assessment, potentially leading to improved intraoperative instrumentation and higher surgical success rates.

Future work consists of system resolution and imaging speed improvements, regular imaging of fresh lumpectomy specimens, and extension to a clinical trial. Also, development of a segmentation technique to differentiate healthy from malignant tissues is needed. Such extensions to the current work will result in a statistical database linking iPAS results to pathology and re-excision surgery rates.

\section{Disclosure}

The authors have no relevant financial interests in the manuscript and no other potential conflicts of interest to disclose.

\section{Acknowledgments}

Ivan Kosik was supported by the Translational Breast Cancer Research Unit (TBCRU) as well as The University of Western Ontario (WGRS). Research funding was provided by the Natural Sciences and Engineering Research Council (NSERC, 312232-2009), the Canadian Institute for Health Research (CIHR, 220298), and the Lawson Health Research Institute (LHRI, LIP). MultiMagnetics Inc. provided support with respect to the development of the data acquisition hardware. This manuscript is an extension of the work presented in Proc. SPIE 10878, Photons Plus Ultrasound: Imaging and Sensing 2019, 108780E (February 27, 2019); 10.1117/ 12.2509552. All studies were carried out with signed informed consent and the approval of the institutional review board of The University of Western Ontario (UWO Research Ethics Board \# 105467; LHSC Tissue Archive \# 888; Lawson Approval \#R-14-311).

\section{References}

1. R. L. Siegel, K. D. Miller, and A. Jemal, "Cancer statistics," CA Cancer J Clin. 65(1), 5-29 (2015).

2. P. Ananthakrishnan, F. L. Balci, and J. P. Crowe, "Optimizing surgical margins in breast conservation," Int. J. Surg. Oncol. 2012, 585670 (2012).

3. M. D. Lagios and M. J. Silverstein, "Ductal carcinoma in situ: recent history and areas of controversy," Breast J. 21(1), 21-26 (2015).

4. M. J. Silverstein and M. D. Lagios, "Treatment selection for patients with ductal carcinoma in situ (DCIS) of the Breast Using the
University of Southern California/Van Nuys (USC/VNPI) Prognostic Index," Breast J. 21(2), 127-132 (2015).

5. J. F. Waljee et al., "Predictors of re-excision among women undergoing breast-conserving surgery for cancer," Ann. Surg. Oncol. 15(5), 12971303 (2008).

6. Canadian Institute for Health Information (CIHI) Breast Cancer Surgery in Canada, 2007-2008 to 2009-2010, Ottawa, ON, CIHI (2012).

7. M. Coates et al., "Beyond the margins-economic costs and complications associated with repeated breast-conserving surgeries," JAMA Surg. 152(11), 2017-2019 (2017).

8. J. L. Vandergrift et al., "Time to adjuvant chemotherapy for breast cancer in national comprehensive cancer network institutions," $105(2)$, 1912 (2013).

9. R. Emmadi and E. L. Wiley, "Evaluation of resection margins in breast conservation therapy: the pathology perspective-past, present, and future," Int. J. Surg. Oncol. 2012, 180259 (2012).

10. K. Esbona, Z. Li, and L. G. Wilke, "Intraoperative imprint cytology and frozen section pathology for margin assessment in breast conservation surgery : a systematic review," Ann. Surg. Oncol. 19(10), 3236-3245 (2012).

11. P. D. Britton et al., "Breast surgical specimen radiographs: how reliable are they?" Eur. J. Radiol. 79(2), 245-249 (2011).

12. M. Thill et al., "MarginProbe final results of the German post-market study in breast conserving surgery of ductal carcinoma in situ," Breast 23(1), 94-96 (2014).

13. H. Eggemann, T. Ignatov, and S. Dan, "Accuracy of ultrasound-guided breast-conserving surgery in the determination of adequate surgical margins," Breast Cancer Res Treat. 145(1), 129-136 (2014).

14. N. M. A. Krekel et al., "Intraoperative ultrasound guidance for palpable breast cancer excision (COBALT trial): a multicentre, randomised controlled trial," Lancet Oncol. 14(1), 48-54 (2013).

15. A. Koloydenko and I. Notingher, "Towards intra-operative diagnosis of tumours during breast conserving surgery by selective-sampling Raman micro-spectroscopy," Phys. Med. Biol. 59(20), 6141-6152 (2014).

16. J. Q. Brown et al., "Optical assesssment of tumor resection margins in the breast," IEEE J. Sel. Top. Quantum Electron. 16(3), 530-544 (2010).

17. N. Lue et al., "Portable optical fiber probe-based spectroscopic scanner for rapid cancer diagnosis: a new tool for intraoperative margin assessment," PLoS One 7(1), e30887 (2012).

18. T. M. Breslin et al., "Autofluorescence and diffuse reflectance properties of malignant and benign breast tissues," Ann. Surg. Oncol. 11(1), 65-70 (2004).

19. F. T. Nguyen et al., "Intraoperative evaluation of breast tumor margins with optical coherence tomography," Cancer Res. 69(22), 8790-8797 (2009).

20. G. R. Kim et al., "Photoacoustic imaging of breast microcalcifications: a preliminary study with 8-gauge core-biopsied breast specimens," PLoS One 9(8), 8-10 (2014).

21. B. Wang, A. Karpiouk, and D. Yeager, "In vivo intravascular ultrasound-guided photoacoustic imaging of lipid in plaques using an animal model of atherosclerosis," Ultrasound Med Biol. 38(12), 2098-2103 (2013).

22. V. S. Dogra et al., "Multispectral photoacoustic imaging of prostate cancer: preliminary ex vivo results," J. Clin. Imaging Sci. 3(3), 1-7 (2015).

23. C. Kim et al., "Deeply penetrating in vivo photoacoustic imaging using a clinical ultrasound array system," Biomed. Opt. Express 1(1), 278-284 (2010).

24. K. H. Song and L. V. Wang, "Deep reflection-mode photoacoustic imaging of biological tissue," J. Biomed. Opt. 12(December), 1-3 (2007).

25. L. V. Wang and S. Hu, "Photoacoustic tomography: in vivo imaging from organelles to organs," Science 335(March), 1458-1462 (2012).

26. R. Li et al., "Assessing breast tumor margin by multispectral photoacoustic tomography," Biomed. Opt. Express 6(4), 1273 (2015).

27. A. Hainsworth et al., "Ductal carcinoma in situ: breast-conserving surgery without radiotherapy," ANZ J. Surg. 85(4), 245-248 (2015).

28. P. Subhedar et al., "Decreasing recurrence rates for ductal carcinoma in situ: analysis of 2996 women treated with breast-conserving surgery over 30 years," Ann. Surg. Oncol. April, 3273-3281 (2015).

29. P. A. Newcomb et al., "Late age at first full term birth is strongly associated with lobular breast cancer," Cancer 117, 1946-1956 (2011). 
30. R. Ellwood et al., "Photoacoustic imaging using acoustic reflectors to enhance planar arrays," J. Biomed. Opt. 19(12), 126012 (2014).

31. M. Jaeger et al., "Deformation-compensated averaging for clutter reduction in epiphotoacoustic imaging in vivo," J. Biomed. Opt. 17(6), 066007 (2012).

32. M. Roumeliotis et al., "Development and characterization of an omnidirectional photoacoustic point source for calibration of a staring 3D photoacoustic imaging system," Opt. Express 17(17), 15228-15238 (2009).

33. M. B. Roumeliotis, I. Kosik, and J. J. L. Carson, "3D photoacoustic imaging using starting, sparse array with 60 transducers," 8223, 82233F (2012).

34. I. Kosik et al., "Combined 3D photoacoustic and 2D fluorescence imaging of indocyanine green contrast agent flow," Proc. SPIE 8581, 1-8 (2013).

35. P. Wong et al., "Objective assessment and design improvement of a staring, sparse transducer array by the spatial crosstalk matrix for 3D photoacoustic tomography," PLoS One 10(4), e0124759 (2015).

36. American National Standard for Safe Use of Lasers. ANSI Z136.1, Washington, DC (2010)

37. M. Xu and L. V. Wang, "Universal back-projection algorithm for photoacoustic computed tomography," Phys. Rev. E 71(1), 16706 (2005).

38. I. S. Sevcenco, P. J. Hampton, and P. Agathoklis, "Seamless stitching of images based on a Haar Wavelet 2D integration method," in IEEE's 17th Int. Conf. on Digital Signal Processing (2011).

39. B. K. P. Horn, "Closed-form solution of absolute orientation using unit quaternions," J. Opt. Soc. Am. A. 4(4), 629 (1987).

40. S. a. Ermilov et al., "Laser optoacoustic imaging system for detection of breast cancer," J. Biomed. Opt. 14(2), 024007 (2009).

41. S. Manohar et al., "Initial results of in vivo non-invasive cancer imaging in the human breast using near-infrared photoacoustics," Opt. Express 15(19), 12277-12285 (2007).

42. Z. Xie et al., "Combined photoacoustic and acoustic imaging of human breast specimens in the mammographic geometry," Ultrasound Med. Biol. 39(11), 2176-2184 (2013).

43. P. G. Anderson et al., "Broadband optical mammography: chromophore concentration and hemoglobin saturation contrast in breast cancer," PLoS One 10(3), 1-23 (2015).

Ivan Kosik received his $\mathrm{PhD}$ in medical biophysics from the Western University, London, Ontario, Canada, in 2018. He received his honors BSc degree in physics from Western University with a specialization in medical physics. Currently, he is a research member of the Lawson Health Research Institute at St. Joseph's Hospital in London, Ontario. $\mathrm{He}$ is also the founder and director of Superior Assembly Inc., a custom assembly services company.
Muriel Brackstone attended Western University, where she received her master's degree in neurosciences in 1994. She attended medical school at UWO and graduated in 1999 (gold medalist). She received her training in general surgery from Western Ontario and became a fellow of the Royal College of Surgeons in 2004. She completed her master's degree in clinical epidemiology and biostatistics at Western University in 2008 followed by a PhD in pathology (clinical trials) at Western University from 2009 to 2014.

Anat Kornecki is an associate professor of medical imaging, head of the Division of Breast Imaging at the Western University and regional breast imaging lead at the South West Regional Cancer Program. Her main area of research is breast imaging.

Astrid Chamson-Reig is a research coordinator with the imaging program at the Lawson Health Research Institute in London, Ontario, Canada. She provides support to the research of Dr. Jeffrey Carson in optics and photoacoustic imaging, conducting the clinical studies and mentoring students in the lab. She is responsible for website management and member support for The Bioelectromagnetics Society, and the online abstract submission system, preparation of conference program and abstract proceedings for BioEM annual meetings.

Philip Wong received his MSc degree in medical biophysics from Western University in 2014. His main area of research is photoacoustic tomography with a focus on system design optimization.

Morteza Heydari Araghi received his BSc degree in electrical and electronics engineering from Amirkabir University of Technology, Tehran, Iran, and his MSc degree in electrical and computer engineering from the University of Western Ontario, London, Canada. Currently, he is affiliated with eVinci Technologies Inc., London, Ontario. His technical experience is in embedded systems, control systems, robotics, instrumentation, automation, and digital image processing.

Jeffrey J. L. Carson is a scientist at the Lawson Health Research Institute and an associate professor at the University of Western Ontario. He is an expert on optical imaging systems. His laboratory specializes in optical device development and has led early developments in snapshot 3-D photoacoustic tomography, snapshot multispectral imaging, and photonic devices for optical imaging and sensing. His experience includes managing large R\&D projects and raising funds for research and commercialization activities. In 2015 he cofounded Spectral Devices Inc. to commercialize pixelated multispectral filter array technology developed in his laboratory. 